\title{
Corner effect and separation in transonic channel flows
}

\author{
P. J. K. BRUCE†, D. M. F. BURTON, N. A. TITCHENER \\ AND H. B ABINSKY \\ Department of Engineering, University of Cambridge, Trumpington Street, Cambridge CB2 1PZ, UK
}

(Received 9 December 2010; revised 22 February 2011; accepted 13 March 2011; first published online 31 May 2011)

An investigation into parameters affecting separation in normal shock wave/boundary layer interactions (SBLIs) has been conducted. It has been shown that the effective aspect ratio of an experimental facility (defined as $\delta^{*} /$ tunnel width) is a critical factor in determining when shock-induced separation will occur. Experiments examining $M_{\infty}=1.4$ and 1.5 normal shock waves in a wind tunnel with a small rectangular cross-section have been performed and show that a link exists between the extent of shock-induced separation on the tunnel centre-line and the size of corner-flow separations. In tests where the corner-flows were modified ahead of the shock (through suction and vortex generators), the extent of separation around the tunnel centre-line was seen to vary significantly. These observations are attributed to the way corner flows modify the three-dimensional shock-structure and the impact this has on the magnitude of the adverse pressure gradient experienced by the tunnel wall boundary layers.

Key words: boundary layer separation, high-speed flow, shock waves

\section{Introduction}

Streamwise corners are a common and often unavoidable feature of many internal transonic flows such as the internal compression intakes for the engines of supersonic aircraft or the working sections of high speed wind tunnels. Often the approach taken in wind tunnel tests is to try and minimise corner effects by focusing measurements on the flow away from the tunnel walls. However, this approach does not guarantee two dimensionality of the flow and there is evidence that the effects of the corner flows cannot be ignored, even on the tunnel centreline. See, for example, the experiments of Chriss et al. (1989) and Titchener \& Babinsky (2011). Furthermore, situations exist where it is not practical to restrict measurements to regions close to the tunnel centreline. This is especially true for small experimental facilities and limits their usefulness as a means for simulating nominally two-dimensional flow-fields.

Flow development in streamwise corners through normal shock wave/boundary layer interactions (SBLIs) can be complex and often the physics of these flows are not fully understood. This lack of understanding represents a major hurdle for the development of numerical methods such as Reynolds-averaged Navier-Stokes (RANS), Large eddy simulation (LES) and detached eddy simulation (DES) codes

$\dagger$ Present address: Department of Mechanical Engineering, Imperial College London. Email address for correspondence: p.bruce@imperial.ac.uk 


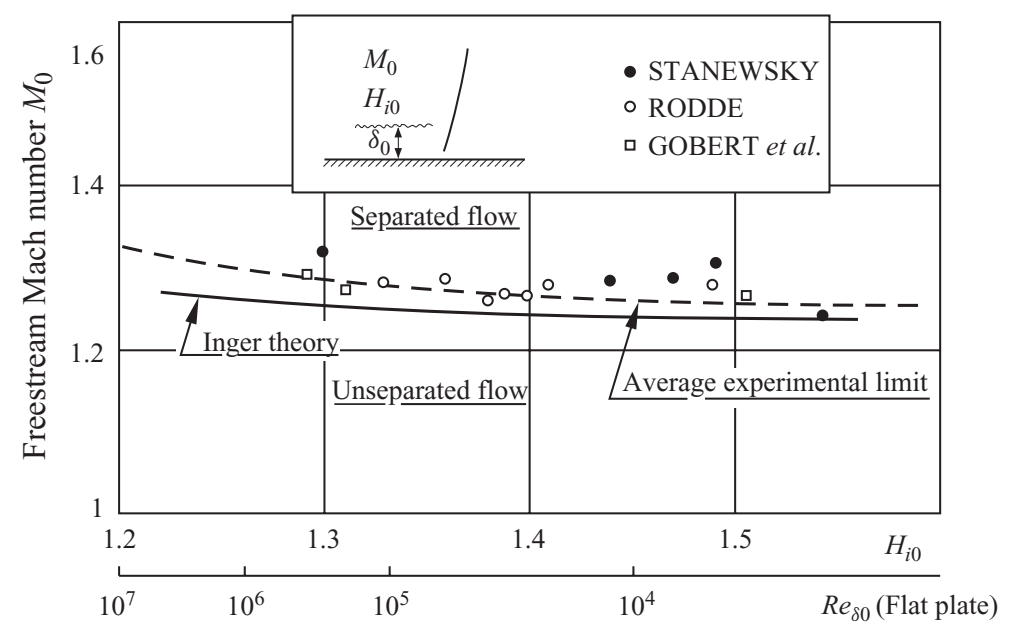

FiguRE 1. Experimental shock-induced incipient separation limit in transonic flow, proposed by Delery (1985).

that are capable of capturing the flow structure in three-dimensional geometries with corners where shock waves are present.

Historically, much research has focused on the prediction of shock-induced separation in normal SBLIs. Principal contributors in this area over the last 60 years include Ackeret, Feldmann \& Rott (1947), Seddon (1960), Kooi (1978), Delery (1985) and Sajben et al. (1991). Researchers agree that interaction strength (specifically the free-stream Mach number ahead of a normal shock wave) is the single most important factor in determining whether a turbulent boundary layer separates or remains attached through a normal SBLI. Other factors such as the incompressible boundary layer shape factor ahead of the interaction $\left(H_{i 0}\right)$ are thought to be of secondary importance. It should also be noted that the more traditionally considered Reynolds number $(R e)$ effect is for the best part incorporated in the incompressible boundary layer shape factor $H_{i 0}$ (these two parameters not being mutually exclusive for a flat plate zero-pressure-gradient boundary layer). This was well illustrated by Delery (1985), who produced the plot shown in figure 1, where the onset of separation is seen to be only weakly dependent on $H_{i 0}$ and occurs at around $M_{\infty}=1.3$ for a wide range of $H_{i 0}$ and $R e$.

The free interaction theory developed by Chapman, Kuehn \& Larson (1957) examines the idea that the flow behaviour up to the point of separation is independent of the post shock geometry, as discussed by Delery (1985) and Zheltovodov, Dvorak \& Safarik (1990). The work of Chapman et al. (1957) was extended by Erdos \& Pallone (1962) to develop a criteria for determining the point at which shock-induced separation occurs. As well as the Mach number ahead of the shock, the skin friction of the incoming flow is also taken into account when calculating the pressure jump that the boundary layer can withstand without separating. However, a limitation of the criteria is that it does not take into account how rapidly the pressure jump occurs due to the interaction length not being considered.

Sajben et al. (1991) also collected data from a number of transonic SBLI experiments that were considered to be nominally two-dimensional and plotted approach Mach number against Reynolds number based on boundary layer momentum thickness $\theta$, see figure 2 . 


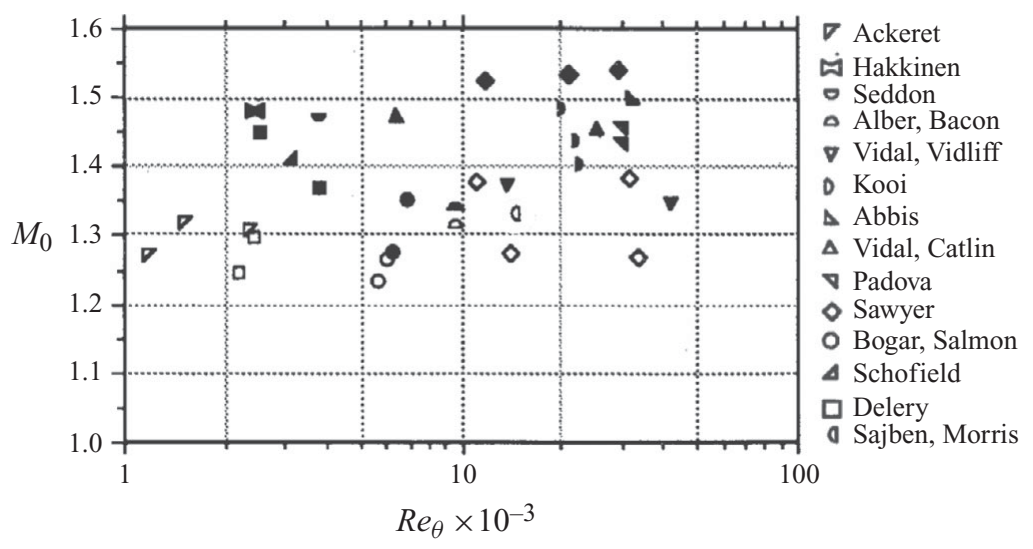

FiguRE 2. Experimental data for attached (open symbols) and separated (filled symbols) transonic SBLIs, from Sajben et al. (1991). The reader is referred to the paper by Sajben et al. (1991) for full details of the references that the experimental data are taken from.

Figure 2 shows that significant variation exists between the Mach numbers corresponding to the onset of shock-induced separation in different experimental facilities. Furthermore, the plot suggests that these differences cannot be attributed solely to differences in $R e$ (or $H_{i 0}$ ).

This paper aims to address this issue and proceeds by reviewing the conditions for shock-induced separation, including re-assessment of the data in figure 2 to demonstrate that three-dimensional effects can also have a strong influence on shockinduced separation. Following this, the results from a range of experiments carried out in Cambridge are presented, with particular emphasis on examining the flow structure in the streamwise tunnel corners.

\section{Review of factors affecting separation in normal SBLIs}

Figures 1 and 2 do not show a clear trend between the onset of incipient separation and flow properties such as $R e$ or $H_{i 0}$ for experimental data from different experimental facilities. The results from a number of experimental studies are plotted in figure 3 against $R e_{\theta}$ and $H_{i 0}$. In addition to the results from figure 2, data are also included from tests by Burton, Babinsky \& Bruce (2010) and Chriss et al. (1989). The latter of these two is particularly noteworthy because they found that the boundary layer along the tunnel centreline remained attached through a $M_{\infty}=1.59$ SBLI in a confined channel. Interestingly, flow visualisation performed for this experiment revealed that extensive flow separation regions were present in the wind tunnel corners. The solid horizontal lines marked in figure 3(a) highlight the lowest Mach number at which separation was observed and also the highest Mach number at which attached flow was maintained on the tunnel floor centreline.

The broken lines marked in figure $3(b)$ correspond to a number of (analytically determined) predicted limits for the onset of incipient separation, including that proposed by Inger (1976) (which also appeared on Delery's plot, see figure 1), Nussdorfer (1956) and Alber et al. (1973). All of the limits shown in figure 3(b) are in reasonable agreement at low $R e$ but exhibit different trends and thus diverge as $R e$ increases.

In the case of the prediction based on free interaction theory, the increase in separation limit with increasing $H_{i 0}$ is due to the strong dependence of the resistance 


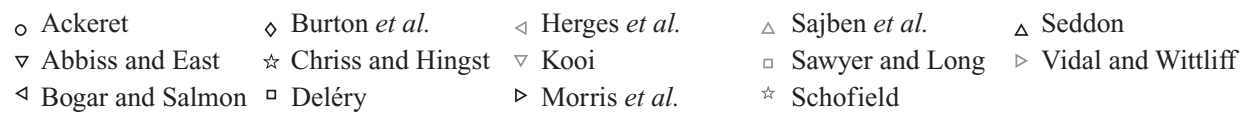

(a)

(b)
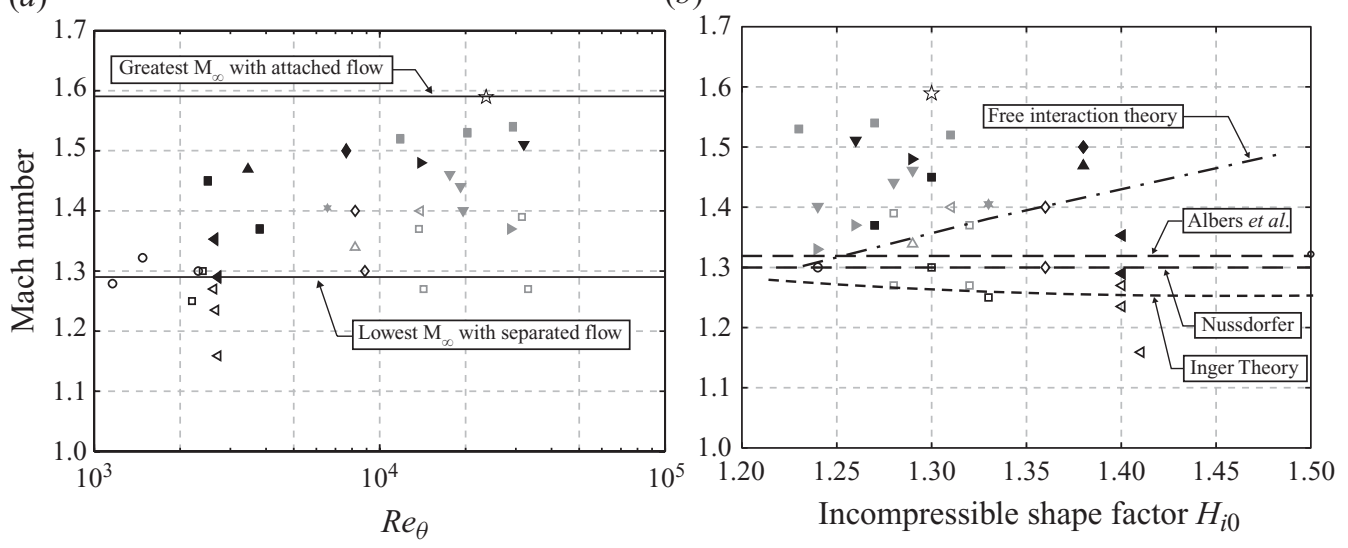

FIGURE 3. Experimental data for attached (open symbols) and separated (filled symbols) transonic SBLIs, plotted against: (a) Reynolds number based on momentum thickness of the incoming boundary layer; $(b)$ incompressible shape factor of the incoming boundary layer.

of the boundary layer to withstand a pressure jump on the skin friction coefficient of the incoming boundary layer, which is essentially a measure of how full the boundary layer velocity profile is. Because a change in $R e$ leads to variation in $\mathrm{C}_{f}$ (see, for example, Van Driest 1951), free interaction theory predicts a significant change in the separation limit with Re. Such a trend is not seen in experiments because it is thought that a higher value of $\mathrm{C}_{f}$ (i.e. a fuller boundary layer profile) in turn leads to a shorter interaction length which strengthens the adverse pressure gradient making separation more likely. This almost exactly cancels the previous effect (greater resistance to separation) making shock-induced separation relatively independent of Reynolds number or shape factor, as discussed by Delery (1985).

Free interaction theory, however, assumes separation onset is observed at a fixed pressure jump, independent of interaction length, thus predicting a strong dependency of separation limit on Re. Inger's theory, in contrast, which takes both effects into account to some extent, actually predicts a slight decrease in the value of $M_{\infty}$ required for separation with increasing $H_{i 0}$. The criteria of Nussdorfer (1956) and Alber et al. (1973) do not incorporate $H_{i 0}$ or $R e$ at all and, therefore, show no variation with separation limits of $M_{\infty}=1.3$ and 1.33, respectively. Crucially though, while some predicted limits are perhaps better than others, none of them can explain the considerable scatter observed in experimental data.

Analysis of the results from flow visualisation studies performed for a number of the experiments in figure 3 highlights an interesting pattern: facilities where separation on the centreline is observed early (e.g. Doerffer \& Szwaba 2004) have relatively small corner separations whereas those where separation on the centreline is seen late or not at all (notably Chriss et al. 1989) have considerable corner separations. Unfortunately, such flow visualisation (or any other information on corner flow topology) is not readily available in the vast majority of the cases shown here. For this reason, we propose to use a new parameter, defined as the ratio of boundary layer displacement thickness in the centre of the tunnel $\left(\delta^{*}\right)$ to tunnel width $(w)$. 


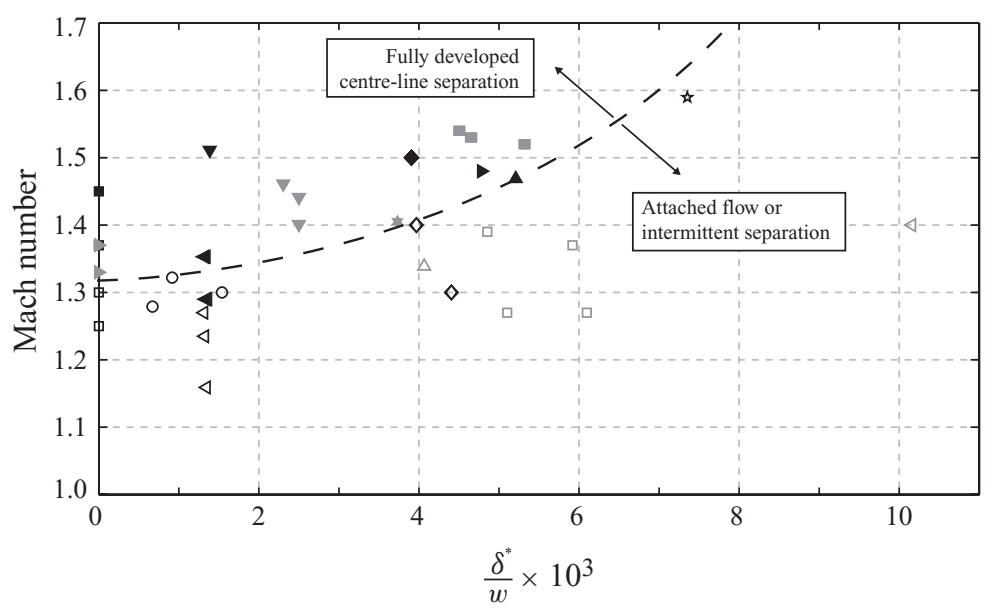

FIGURE 4. Experimental data for attached (open symbols) and separated (filled symbols) transonic SBLIs, plotted against the variable $\delta^{*} / w$. Symbols are as defined in figure 3.

While not being an exact quantifier of corner separation size, it is thought that the extent of corner flow separation should scale (at first order) with $\delta^{*}$, so that $\delta^{*} / w$ can be seen as an estimate of the three-dimensionality of a particular flow. Values of $\delta^{*} / w$ close to zero correspond to experiments that are nominally two-dimensional (such as those in axisymmetrical facilities or those in large facilities with thin boundary layers) while large values of $\delta^{*} / w$ represent more highly confined experiments in small facilities with relatively thick boundary layers. The results in figure 3 have been re-plotted in figure 4 using this new parameter.

Figure 4 shows that the use of the variable $\delta^{*} / w$ enables the results from a large number of different experimental facilities to be divided into two regions (with and without separation) with only a single exception. This is in contrast to the scatter seen previously in the plots in figure 3, which suggests that geometrical differences between experimental facilities are a significant factor that can affect the onset of separation. Furthermore, the ratio of displacement thickness to tunnel width appears to be a suitable variable for comparing the results from different facilities.

Interestingly, the $M_{\infty}=1.4$ data point (attached flow) from Burton et al. (2010) lies very slightly below the dashed line in figure 4, suggesting that it may be sensitive to manipulations of the corner flow-field. In practice, any reduction of the corner flow separation should push the test case towards the domain where flow separation in the centre is observed. This idea is explored in $\S 3$ of this paper.

\section{The relationship between corner flows and the onset of shock-induced boundary} layer separation

It is hypothesised that the corner flows within a wind tunnel working section can have a significant impact on the entire flow-field, including the centreline, where regions of separated flow may be present. Two possible mechanisms for how corner flows affect the centreline are proposed: Firstly, large corner flow separations may cause a large three-dimensional bifurcation of the shock wave structure that spans a significant proportion of the wind tunnel and has an effect on the amount of pressure smearing experienced by the tunnel wall boundary layers. This is in agreement with the findings of previous authors such as Weber et al. (2002) and Handa, Masuda \& Matsuo (2005), who observed that the 3-D (bifurcated) shock structure produced in a 


$\begin{array}{ccccc}M_{\infty} & \delta(\mathrm{mm}) & \delta^{*}(\mathrm{~mm}) & \theta(\mathrm{mm}) & \mathrm{H} \\ 1.4 & 4.9 & 0.86 & 0.41 & 2.04(1.36) \\ 1.5 & 4.1 & 0.85 & 0.37 & 2.28(1.37)\end{array}$

TABLE 1. Compressible integral boundary layer parameters for incoming flow, measured at $x=-30 \mathrm{~mm}$ on the tunnel centreline using LDA. Incompressible shape factor is given in parentheses.

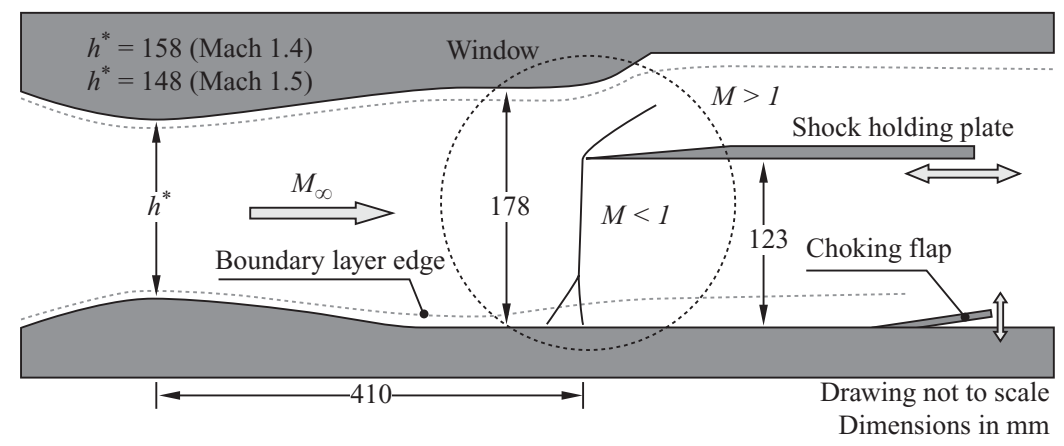

FIGURE 5. Schematic diagram of wind tunnel setup.

confined transonic SBLI increased the amount of pressure smearing near the corners. Secondly, corner flow separations may act as an effective blockage to the core flow in the tunnel and (depending on their size and relative positions) could decelerate the (supersonic) pre-shock flow and/or re-accelerate the (subsonic) post-shock flow, both of which would act to smear the adverse pressure gradient imposed by the normal shock wave.

To further investigate whether both of the mechanisms described above are plausible, experiments have been conducted in the Cambridge University supersonic wind tunnel facility. Two sets of experiments have been performed to assess the impact of corner flows on the appearance of shock-induced separation: firstly, suction was used to reduce the size of the corner flow separations in a $M_{\infty}=1.4$ normal SBLI, where the boundary layer on the tunnel floor centreline is normally attached. Secondly, vortex generators were used to modify the corner flows upstream of a $M_{\infty}=1.5$ normal SBLI, where the boundary layer on the tunnel floor centreline is normally separated and moderate size corner flow separations are present.

\section{Experiments studying the effects of manipulating the corner flows}

\subsection{Methodology}

The supersonic wind tunnel at the University of Cambridge has a rectangular parallelwalled working section with a constant cross-section $114 \mathrm{~mm}$ wide by $178 \mathrm{~mm}$ high. A schematic diagram of the working section is depicted in figure 5. Two pairs of interchangeable convergent-divergent nozzle blocks were used to set the Mach number in the working section to $M_{\infty}=1.4$ and 1.5. The wind tunnel is a blowdown facility and tunnel run times of approximately $35-40 \mathrm{~s}$ were possible with the arrangement shown. Properties of the incoming boundary layer with $M_{\infty}=1.4$ and 1.5 are provided in table 1 . These values were calculated from velocity measurements on the tunnel centreline obtained using laser Doppler anemometry (LDA). 

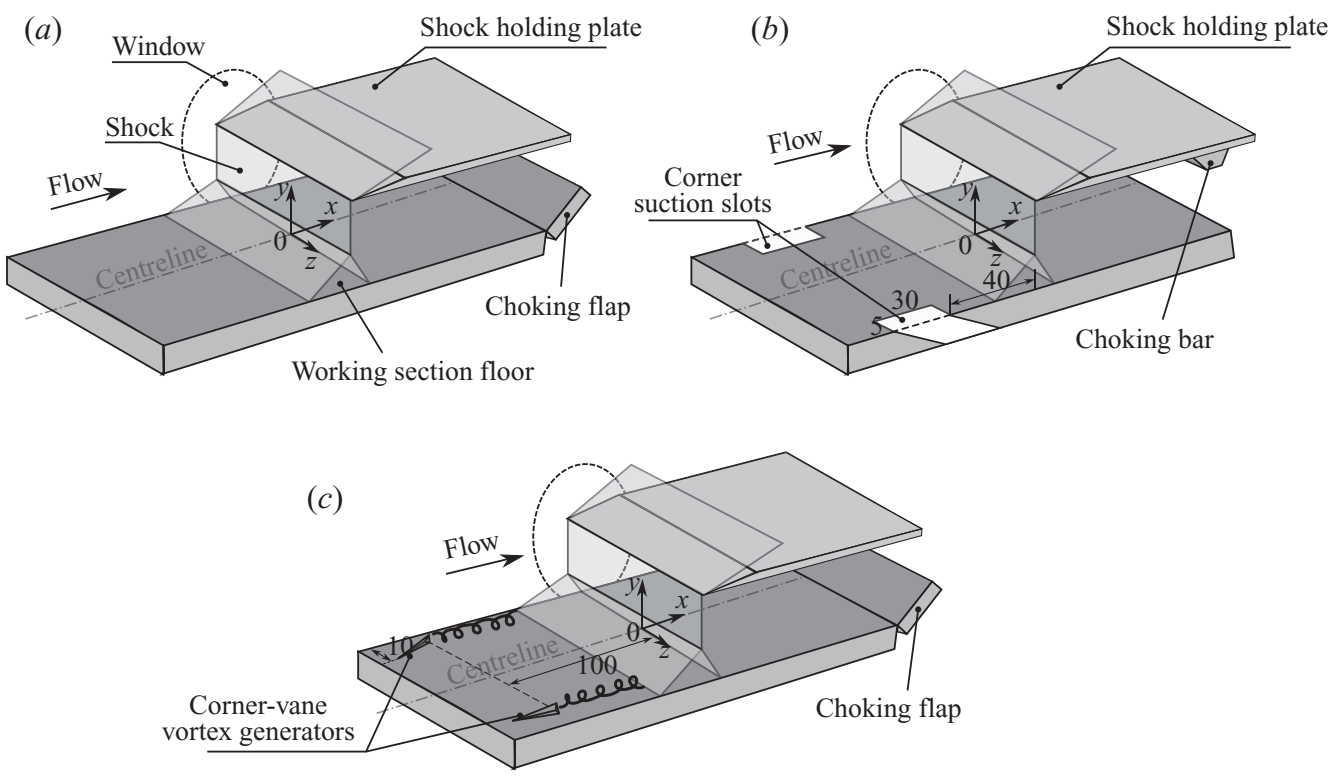

FIGURE 6. Schematic diagrams showing the experimental arrangements used for tests manipulating the corner flows: $(a)$ no-control case; $(b)$ suction experiments; $(c)$ corner-VG experiments.

Flow patterns on the wind tunnel floor and sidewalls were studied using the technique of surface oil flow visualisation. This involved coating the wind tunnel walls with a mixture of white titanium dioxide powder, paraffin and oleic acid before tunnel runs and photographing the resulting flow patterns once the tunnel run had finished. Information on the pressure distribution in the interaction region was obtained through pressure measurements made using a 16-channel Scanivalve Netscanner 9116 pressure system and also with pressure sensitive paint (PSP). Pressure measurements made with the Netscanner system have an accuracy of better than $\pm 0.2 \%$. Pressures obtained using the PSP system have an uncertainty of approximately $\pm 1 \%$. Schlieren photography was used to visualise the interaction structure.

In order to examine the effect of modifying the corner flow, experiments were conducted using active suction through slots in the corners on the floor of the wind tunnel working section upstream of the shock wave. During the suction experiments, the mass flow through the suction system was $0.2 \%$ of the mass flow through the wind tunnel working section. Experiments were also conducted with vortex generators (VGs) placed in the tunnel corners upstream of the shock. The VGs used were a vane-type design with a maximum height of $2 \mathrm{~mm}(h / \delta \approx 0.4)$ and length $20 \mathrm{~mm}$. Details of the experimental arrangements for these tests are shown in figure 6.

\section{2. $M_{\infty}=1.4$ experiments}

The $M_{\infty}=1.4$ baseline oil flow is shown in figure $7(a)$. The approximate extent of the regions influenced by separation in the tunnel corners are indicated by a dashed line, a dot-dashed line represents regions of separated flow away from the corners and a solid black line indicates the nominal shock location. At this Mach number, there is no clear separation at the centre of the tunnel floor although slight separation is seen along the sidewalls. The supersonic Mach number of the incoming flow implies that the deflection of the streamlines away from the corners ahead of the shock wave 
(a)

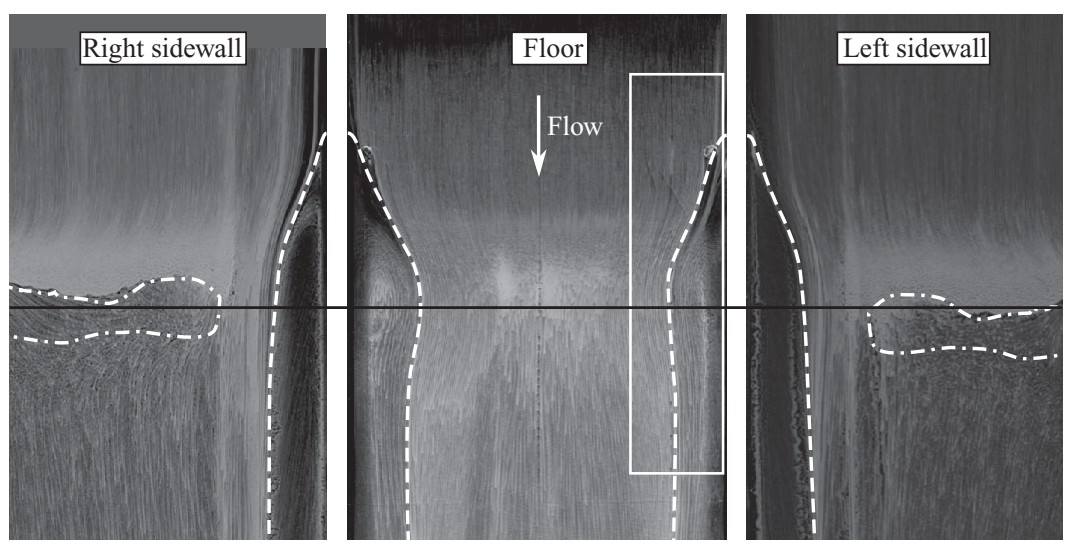

(b)
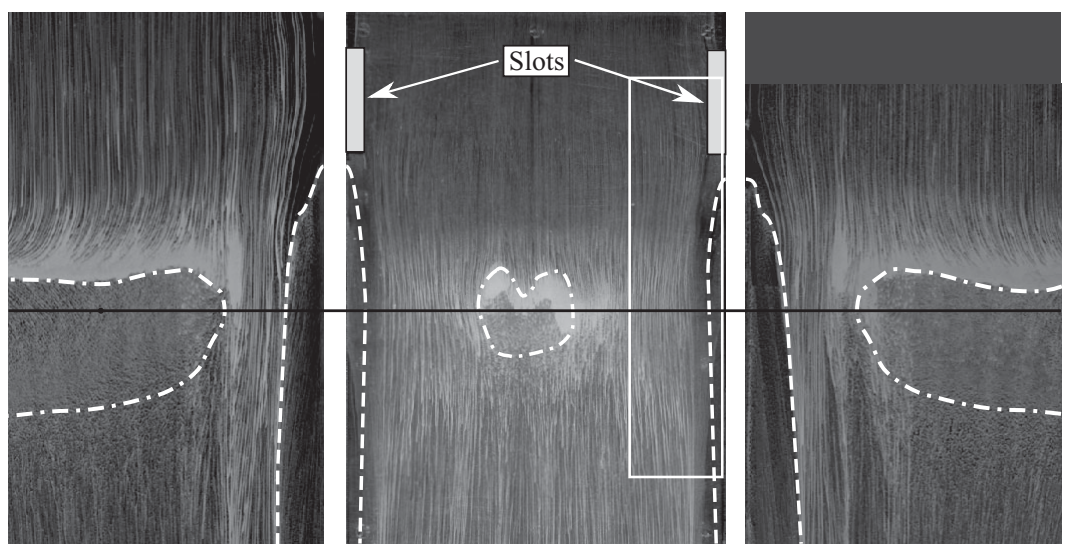

FIGURE 7. Oil flow patterns on the tunnel floor and sidewalls for a $M_{\infty}=1.4$ shock: $(a)$ baseline flow and $(b)$ with corner suction. Only the bottom half of the tunnel sidewalls are shown $(0<y<89 \mathrm{~mm})$.

must be caused by additional compression waves. These compression waves must decelerate the flow in the corner regions, thus reducing the local strength of the main (supposedly 'normal') shock wave in the corners. This effect is likely to be especially significant in small facilities (large $\delta^{*} / w$ ), where the corner separations are relatively large.

The $M_{\infty}=1.4$ oil flow visualisation with corner suction is provided in figure $7(b)$. It can be seen clearly that the regions influenced by separation in the tunnel corners are greatly reduced in size while a separation region appears along the centreline of the tunnel floor. The sidewall separation regions also appear to have grown in size. Figure 8 shows an enlarged image of the regions highlighted by the white boxes in figure 7. It is clear that the suction has resulted in a reduction in the size of the separated flow region in the tunnel corners.

Figure 9 shows schlieren images of the $M_{\infty}=1.4$ SBLI with and without corner suction. Both images show similar SBLI structures, albeit with some subtle differences. In the baseline flow, a strong compression fan exists upstream of the main shock. When suction is applied, this compression fan is replaced with a more clearly defined oblique shock to produce a classic $\lambda$-shock-foot structure, with a relatively weak rear 


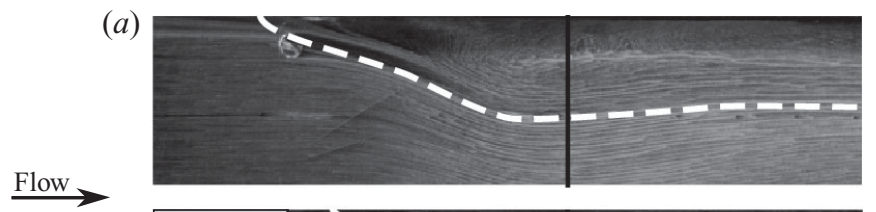

(b)

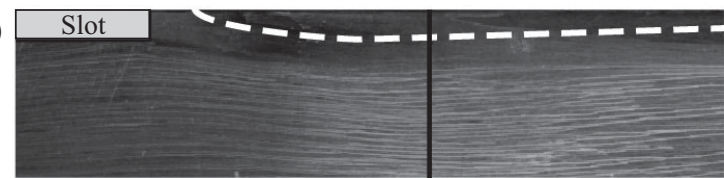

FiguRE 8. Zoom of oil flow patterns in the tunnel corners at $M_{\infty}=1.4:(a)$ baseline flow and (b) with corner suction.

(a)

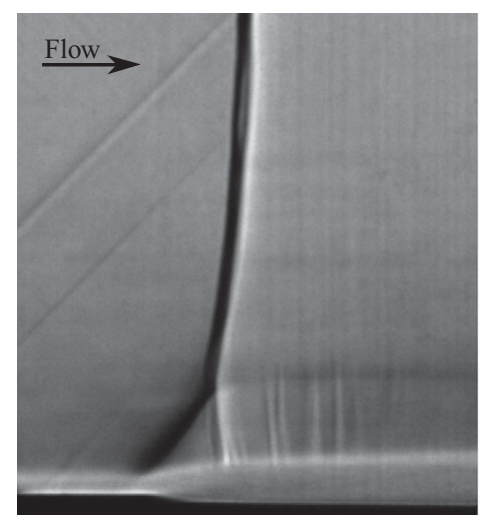

(b)

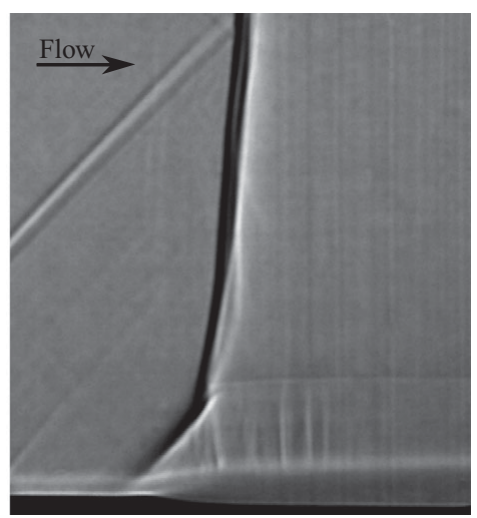

FIGURE 9. Schlieren comparison between $M_{\infty}=1.4$ interaction: $(a)$ baseline case and $(b)$ with corner suction. Only the bottom half of the tunnel is shown $(0<y<89 \mathrm{~mm})$.

leg. This structure is linked to the onset of shock-induced separation, as explained by many previous researchers, such as Delery (1985) and Atkin \& Squire (1992).

Figure 10 compares the pressure rise through the shock with and without suction at $M_{\infty}=1.4$. The pressures were measured along the tunnel floor centreline. It can be seen that suction produces a slight drop in pressure ahead of the shock and a reduced upstream influence, which leads to a sharper pressure rise through the initial part of the SBLI (at around $x=-12 \mathrm{~mm}$ ). This increased pressure gradient is consistent with the formation of the $\lambda$-shock foot structure in figure $9(b)$ and is the likely cause of the centreline separation seen in figure $7(b)$.

Figure 11 shows the pressure distribution on the wind tunnel floor obtained with PSP for the baseline and suction experiments at $M_{\infty}=1.4$. Regions of low pressure can be seen emanating from the slots on either side of the tunnel floor in figure $11(b)$. These are expansion fans created by the effective convex surface curvature produced by suction of the boundary layer. The mass flow removed by the suction slots was calculated to be $0.2 \%$ of the total tunnel mass flow. At the streamwise location of the slots (upstream of the shock), the removal of this amount of low momentum fluid is estimated to increase the effective cross-sectional area of the tunnel by approximately $0.5 \%$. One-dimensional compressible flow theory predicts that this should increase the 'average' Mach number in the tunnel from 1.4 to 1.407 (and hence decrease the pressure ratio $p / p_{0}$ from 0.314 to 0.311 ). 


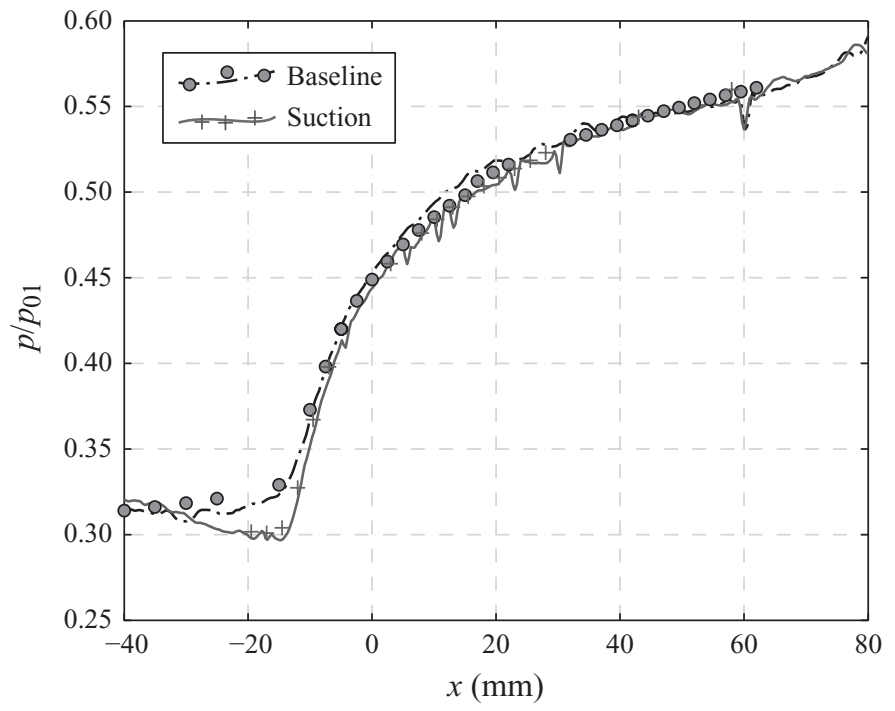

FiguRE 10. $M_{\infty}=1.4$ static pressure measurements along tunnel floor centreline $(z=0)$ for baseline and suction. Symbols correspond to measurements made using pressure tappings. The dashed and solid lines were obtained from tests using pressure sensitive paint

(a)

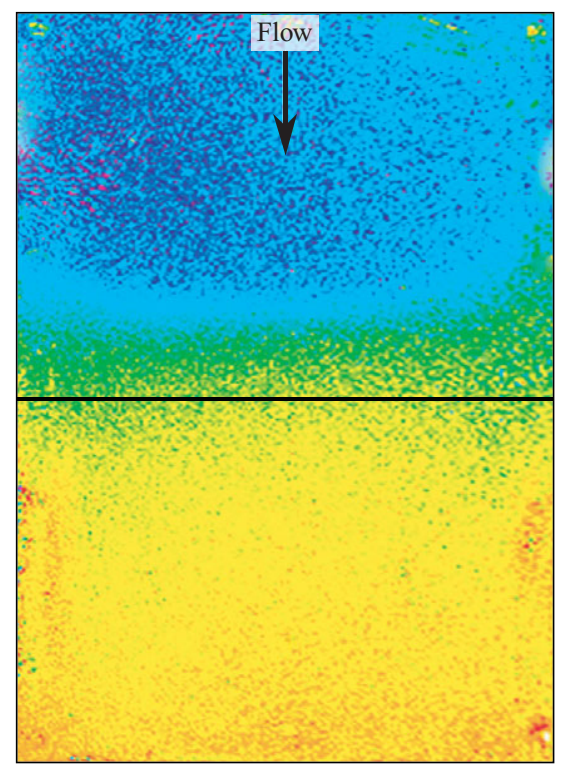

(b)

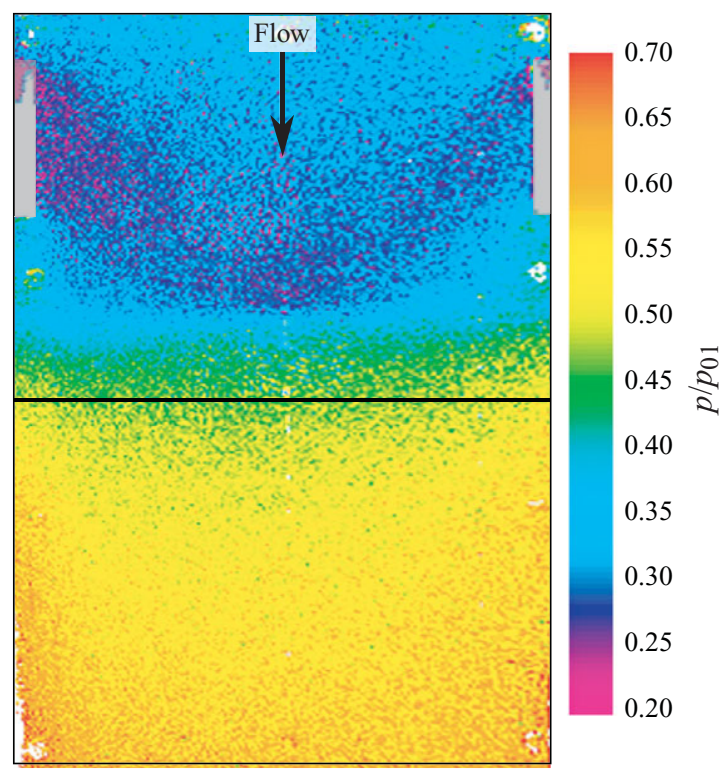

FIGURE 11. (Colour online available at journals.cambridge.org/FLM) Static pressure distribution on the tunnel floor with $M_{\infty}=1.4$ normal shock: $(a)$ baseline; $(b)$ with corner suction. Flow is from top to bottom. Both images show the full tunnel width $(-57<z<57 \mathrm{~mm})$. The nominal shock position $(x=0)$ is shown by the continuous line.

Figure 10 confirms that suction causes the pressure ahead of the shock to decrease, although the minimum value reached is approximately 0.30 , rather than 0.311 . This discrepancy can be explained by the way the (three-dimensional) expansion fans produced by the suction slots meet on the tunnel centreline a short distance upstream 


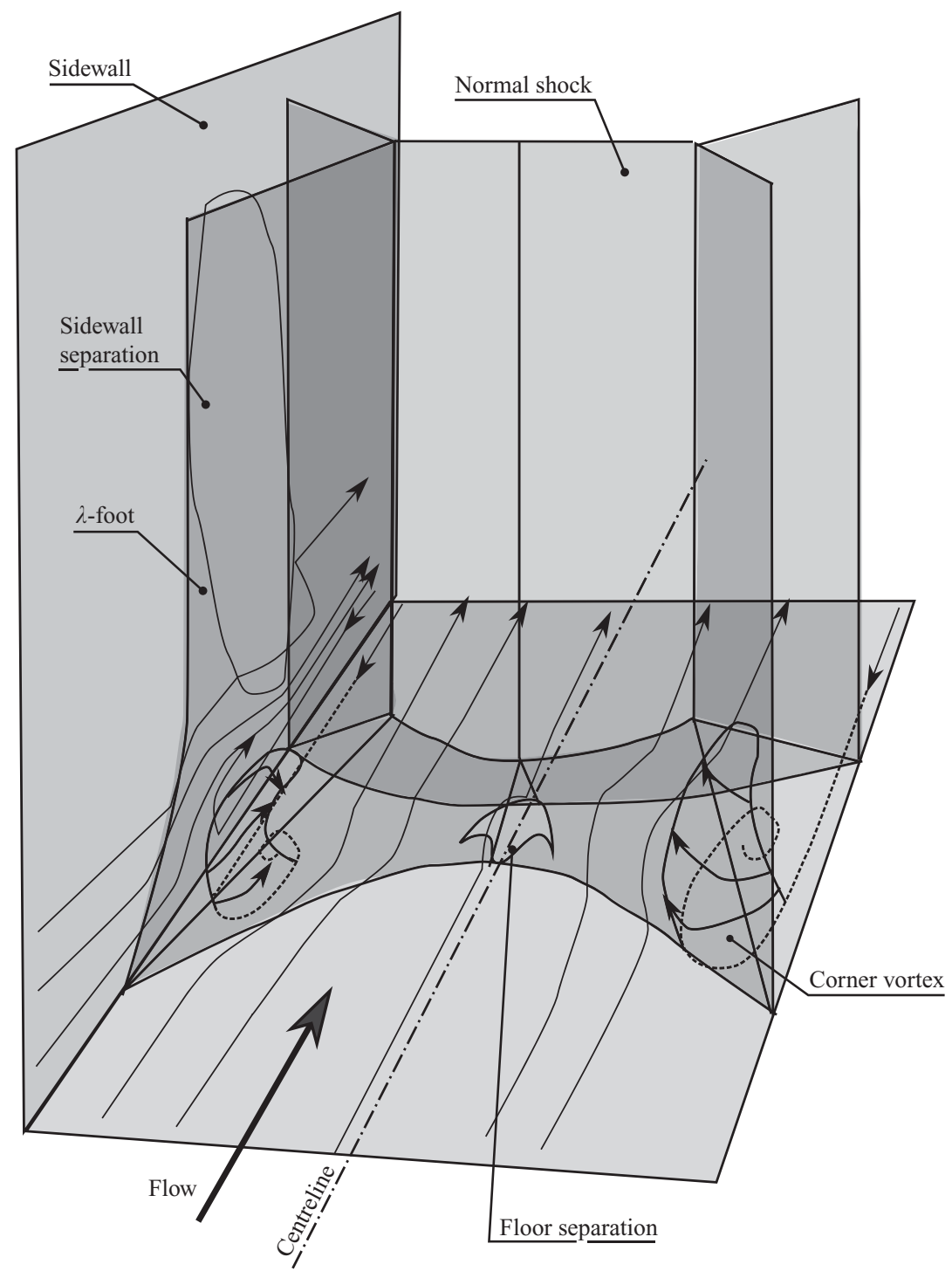

FiguRE 12. Proposed three-dimensional bifurcated shock wave structure in a non-square rectangular channel with significant corner separations.

of the normal shock to produce a region where the flow is locally over-expanded to a Mach number of approximately 1.43. It is likely that this modest increase in local Mach number around the centreline may also have some impact on the appearance of separation there.

The baseline PSP in figure 11(a) shows that the amount of streamwise pressure smearing either side of the tunnel centreline is greater than it is on the centreline. This increase in pressure smearing near the tunnel corners is caused by an increase in the upstream influence of the SBLI. This observation suggests that the separated flow regions in the tunnel corners cause an increase in the size of the bifurcated shock structure in the corner regions. This proposed mechanism is illustrated in figure 12. Figure 11(b) shows that corner suction decreases the amount of pressure smearing 

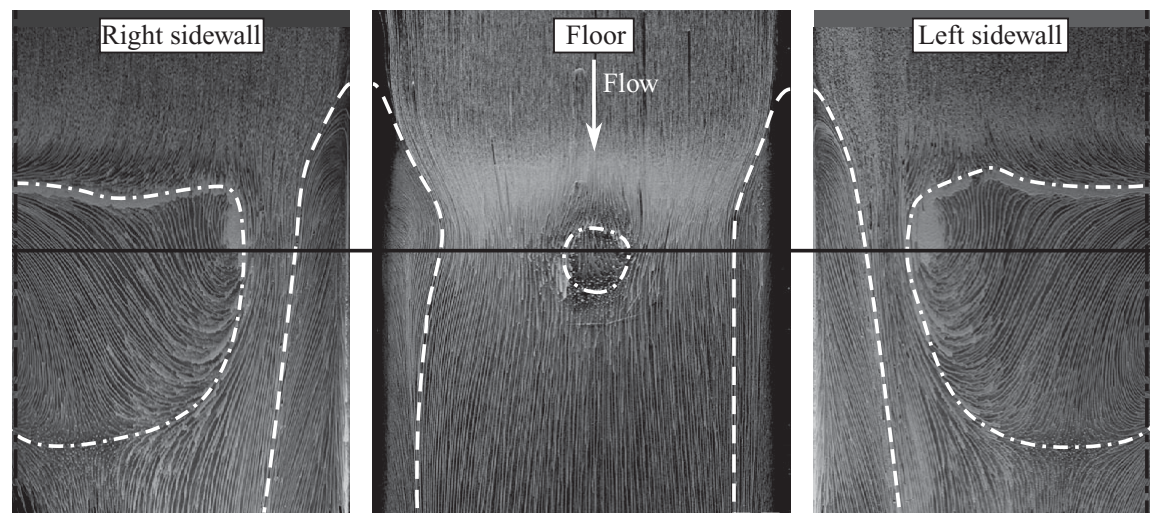

FIGURE 13. Oil flow patterns on the tunnel floor and sidewalls for a $M_{\infty}=1.5$ shock. Only the bottom half of the tunnel sidewalls are shown $(0<y<89 \mathrm{~mm}$.)

across the entire tunnel span, which would be consistent with the absence or reduction of this effect.

Figure 12 highlights a significant difference between the flow structure in regions of separation on the tunnel floor centreline and in the corners. Separation on the centreline is relatively two-dimensional, with a so-called bubble of reversed flow contained between well-defined separation and reattachment lines. In contrast, the separated regions in the tunnel corners are highly three-dimensional with strong vortex formation, no clear regions of reversed flow and without a well-defined point or line of re-attachment. The flow in-between the separation bubble at the centreline and the corner vortices is attached.

The experiments presented thus far with $M_{\infty}=1.4$ have explored the idea of increasing likelihood of separation in the centre when corner separation is reduced. In the following, we consider another example where the same relationship between separation in the centre and in the corners is observed. This test case is a stronger $M_{\infty}=1.5$ normal SBLI, where (unlike at $M_{\infty}=1.4$ ) the flow is separated along the centreline in the absence of any corner flow modifications.

\section{3. $M_{\infty}=1.5$ experiments}

The oil flow in figure 13 shows that the $M_{\infty}=1.5$ baseline flow has a separated region along the centreline of the tunnel floor. Clear separation regions are also apparent on the sidewalls.

The introduction of the corner vanes in the $M_{\infty}=1.5$ flow resulted in a substantial increase in the size of the separated regions in the tunnel corners, which can be clearly seen in figure 14. In contrast, there is no indication of shock-induced separation on the centreline of the tunnel floor. A possible explanation for this behaviour is that the vortices produced by the VGs induce a down-wash in the centre of the tunnel, which makes the boundary layer there more robust. However, this is unlikely because of the large span-wise distance between the VG location and the tunnel floor centreline.

The schlieren images in figure 15 suggest that there may be small differences between the size of the $\lambda$-foot in each test case, although the images are not conclusive. Both images show a broadly similar level of boundary layer growth through the interaction. Image $(a)$ shows the existence of a faint shear-layer that emanates from the triple point, while $(b)$ does not. The leading shock-leg in image $(b)$ also appears to be slightly weaker and at a more normal angle than in the baseline test case. These observations 


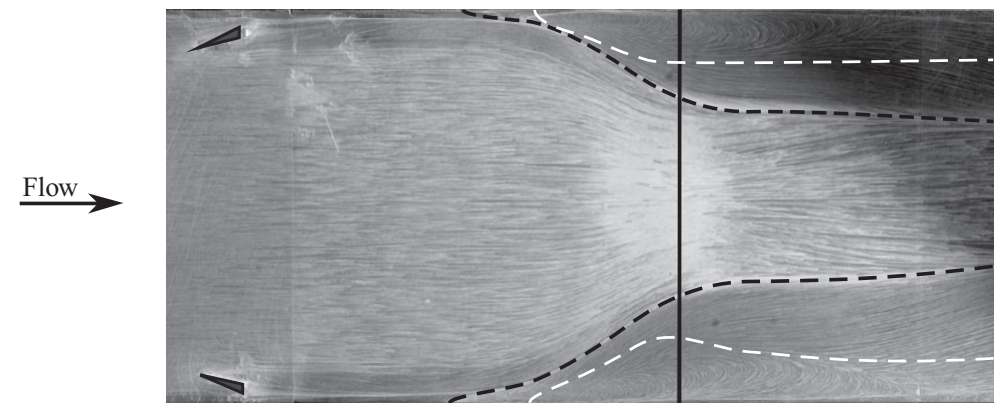

FIGURE 14. Oil flow pattern on the tunnel floor with VGs placed in the corners upstream of a $M_{\infty}=1.5$ shock. The dashed white lines show the approximate extent of the separated regions in the tunnel corners for the baseline test case for comparison.

(a)

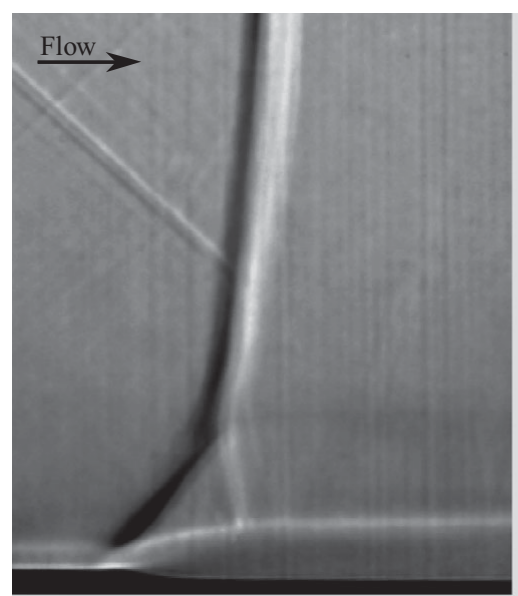

(b)

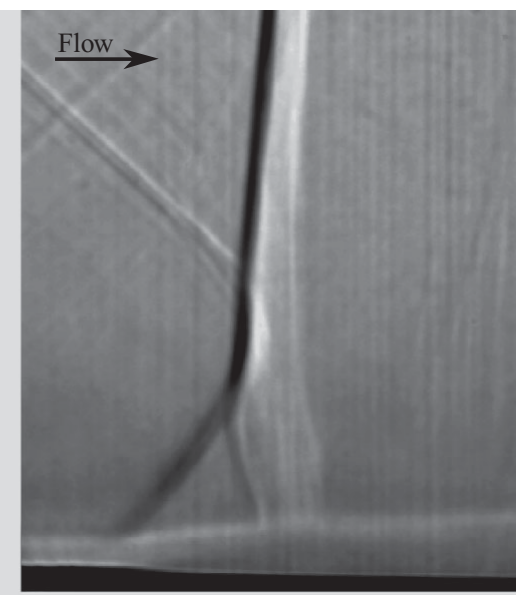

FIGURE 15. Schlieren images of the $M_{\infty}=1.5$ interaction: $(a)$ baseline case; $(b)$ with corner vanes. Only the bottom half of the tunnel is shown $(0<y<89 \mathrm{~mm})$.

are consistent with an interaction of reduced strength without separation, in agreement with the oil flows.

Figure 16 contains information on the streamwise pressure gradient on the tunnel floor at $M_{\infty}=1.5$ with and without corner-vane VGs. The pressure profiles show that the addition of corner vanes reduces the adverse pressure gradient on the tunnel floor centreline. This is consistent with the disappearance of separation observed in the oil flow when corner-vane VGs were used at $M_{\infty}=1.5$. This result further supports the idea that separations in the corners and on the centreline of an experimental facility are related through the way in which features of the flow modify the streamwise pressure gradients experienced by the boundary layers in different parts of the facility.

In the case of the $M_{\infty}=1.5$ test case described here, the reduced adverse pressure gradient with corner-vane VGs is due to a combination of an increased upstream influence and reduced downstream pressure. The increase in upstream influence is attributed to the way the corner separations modify the bifurcated shock structure, while the lower downstream pressure is attributed to re-acceleration of the post-shock 


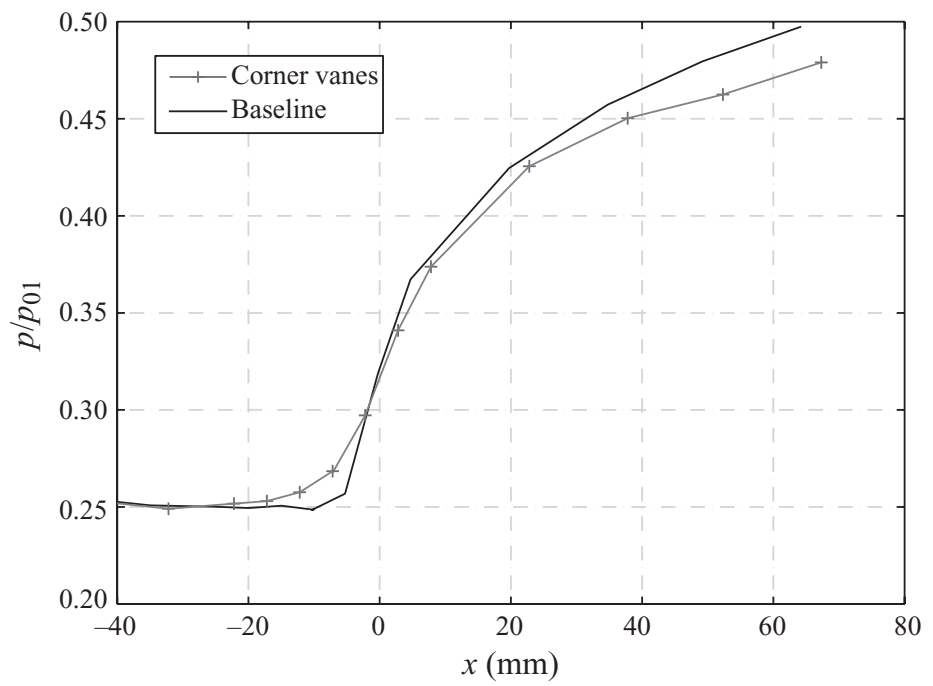

FIGURE 16. $M_{\infty}=1.5$ static pressure measured on the tunnel centreline in the baseline and with corner-vane VGs.

core-flow due to the blockage effect from the large regions of separation in the corners. This blockage effect is supported by the oil flow visualisation in figure 14, which shows that the regions of separation in the corners are much larger downstream of the shock in the test case with corner-vane VGs. The schlieren images with and without the corner-vane VGs in figure 15 provide some evidence that the corner-vane VGs do slightly increase the upstream influence of the SBLI.

\section{Conclusions}

Previously proposed limits for the onset of separation in normal SBLIs based on experimental or analytical results give reasonable agreement in facilities where the flow is considered to be nominally two-dimensional. However, results from different experimental facilities show large discrepancies that are not predicted by previous theories.

In the present study, it has been found that when a tunnel confinement ratio is introduced the results fall into a much clearer trend. More 'three-dimensional' facilities, where the ratio of $\delta^{*}$ and $w$ is large show a delay of classical quasi-2-D shock-induced separation on the centreline. The parameter $\delta^{*} / w$ seems to capture the relative importance of three-dimensional effects (including the influence of regions of separation in the tunnel corners) on the flows in different facilities.

This result supports the findings of previous studies that have suggested a link between corner flows and centreline separation may exist. This includes experimental studies of supersonic shock reflection SBLIs, such as those performed by Dupont et al. (2005) and Doerffer et al. (2010), where the authors also report that the extent of the shock-induced separation region on the tunnel floor is strongly affected by the state of the flow in the tunnel corners and on the sidewalls. This suggests that there may be significant similarities between transonic and supersonic SBLIs in this respect.

Experiments manipulating the corner flows upstream of a $M_{\infty}=1.4$ normal SBLI using corner suction have shown the possibility of introducing a separated region in 
a previously attached flow-field by reducing the size of corner effects. A suction mass flow rate of $0.2 \%$ of the total wind tunnel mass flow was used to achieve this result.

Additional experiments examining a $M_{\infty}=1.5$ shock were conducted. Here, the incoming flow was manipulated to give an exaggerated corner effect and this eliminated shock-induced separation on the tunnel centreline. A mechanism which explains this trend has been suggested based on the relationship between large corner flow separations and variations in the shock-induced streamwise adverse pressure gradient across the tunnel span. These results raise questions over assumptions that the vast majority of experiments can be treated as 'nominally two-dimensional'.

The authors gratefully acknowledge the support of part of this work by AFRL (Grant No. FA8655-08-1-3091)

\section{REFERENCES}

Ackeret, J., Feldmann, F. \& Rott, N. 1947 Investigations of compression shocks and boundary layers in gases moving at high speed. NACA Tech. Rep. TM1113.

Alber, I. E., Bacon, J. W., Masson, B. S. \& Collins, D. J. 1973 An experimental investigation of turbulent transonic viscous-inviscid interactions. AIAA J. 11 (5), 620-627.

AtKin, C. J. \& SQuire, L. C. 1992 A study of the interaction of a normal shock wave with a turbulent boundary layer at Mach numbers between 1.30 and 1.55. Eur. J. Mech. 11 (1), 93-118.

Burton, D. M. F., Babinsky, H. \& BruCE, P. J. K. 2010 Experimental investigation into parameters governing corner interactions for transonic shock-wave/boundary-layer interactions. AIAA Paper 2010-0871.

Chapman, D. R., Kuehn, D. M. \& Larson, H. K. 1957 Investigation of separated flow in supersonic and subsonic streams with emphasis on the effects of transition. Technical note 3869. NACA, Washington.

Chriss, R. M., Hingst, W. R., Strazisar, A. J. \& Keith, T. G. 1989 An LDA investigation of threedimensional normal shock wave boundary-layer interaction. In Proc. Transonic Symposium: Theory, Application and Experiment, vol. 1, Part 2, pp. 741-764. NASA Langley Research Center.

Delery, J. 1985 Shock wave/turbulent boundary layer interaction and its control. Prog. Aerosp. Sci. 22 (4), 209-280.

Doerffer, P., Hirsch, C., Dussauge, J.-P. \& Babinsky, H. (Ed.) 2010 Unsteady Effects of Shock Wave induced Separation: 220 (Notes on Numerical Fluid Mechanics and Multidisciplinary Design), 1 st edn. Springer.

DoerfFer, P. \& SzWABA, R. 2004 Shock wave boundary layer interaction control by streamwise vortices. In Proc. XXI ICTAM 15-21 August, Warsaw, Poland.

Dupont, P., Haddad, C., Ardissone, J.-P. \& Debieve, J. F. 2005 Space and time organisation of a shock wave/turbulent boundary layer interaction. Aerosp. Sci. Technol. (9), 561-572.

Erdos, J. \& Pallone, A. 1962 Shock-boundary layer interaction and flow separation. In Proc. Heat Transfer and Fluid Mechanics Institute, pp. 239-254.

Handa, T., Masuda, M. \& Matsuo, K. 2005 Three-dimensional normal shock-wave/boundary-layer interaction in a rectangular duct. AIAA J. 43 (10), 2182-2187.

INGER, G. R. 1976 Analysis of transonic normal shock-boundary layer interaction and comparisons with experiments. Tech. Rep. Virginia Inst. and State Univ.

KooI, J. W. 1978 Influence of free-stream Mach Number on Transonic Shock-Wave Boundary-Layer Interaction. MP Tech. Rep. 78013 U. National Aerospace Laboratory NLR.

NusSDORFER, T. J. 1956 Some observations of shock-induced turbulent separation in supersonic diffusers. Tech. Rep.

Sajben, M., Morris, M. J., Bogar, T. J. \& Kroutil, J. C. 1991 Confined normal-shock/turbulentboundary-layer interaction followed by an adverse pressure gradient. AIAA J. 29 (12), 21152123. 
SEDDON, J. 1960 The flow produced by interaction of a turbulent boundary layer with a normal shock wave of strength sufficient to cause separation. Tech. Rep. 3502. Ministry of Technology ARC RM.

TitcheneR, N. \& BABINSKy, H. 2011 Micro-vortex generators applied to a flow-field containing a normal shock-wave and diffuser. AIAA J. 49 (5), 1046-1056.

VAn Driest, E. R. 1951 Turbulent boundary layer in compressible fluids. J. Aeronaut. Sci. 18 (3), $1-20$.

Weber, A., Schreiber, H.-A., Fuchs, R. \& Steinert, W. 2002 3-D Transonic flow in a compressor cascade with shock-induced corner stall. J. Turbomach. 124, 358-366.

Zheltovodov, A., Dvorak, R. \& Safarik, P. 1990 Shock wave/turbulent boundary layer interaction properties at transonic and supersonic speed conditions. Izvestiya SO AN SSSR 6, 31-42. 